\title{
Beeinflusst die pulmonale Medikation den Glukosestoffwechsel?
}

\author{
Der ärztliche Alltag zeigt, dass eine hohe Komorbidität zwischen COPD und \\ Typ-2-Diabetes besteht. Das pathogenetische Bindeglied zwischen den beiden \\ Erkrankungen dürfte die systemische Inflammation sein. Besonders relevant \\ ist die Frage, ob und wie stark durch die Therapie der COPD der Glukosestoff- \\ wechsel beeinflusst wird.
}

\begin{abstract}
— „Die COPD ist keinesfalls nur eine Lungenerkrankung, sondern vielmehr ein systemischer Entzündungsprozess", sagte Prof. Claus Franz Vogelmeier, Marburg. Der prädominante Faktor bei der Pathogenese der Erkrankung ist ein Entzündungsprozess in den kleinen Atemwegen. Im weiteren Verlauf kommt es zu einem „spill over“, d. h. der Entzündungsprozess verlässt die Bronchien und greift auf den gesamten Organismus über.
\end{abstract}

\section{Assoziation oder Kausalität?}

Unbestritten ist, dass die COPD mit extrapulmonalen Symptomen einhergeht. Dazu gehören insbesondere die Kachexie, die Muskelatrophie, die Osteoporose, die KHK und schließlich das metabolische Syndrom bzw. der Typ-2-Diabetes. Vieles spricht dafür, dass insbesondere bei der Manifestation der KHK der systemische, durch die COPD induzierte Entzündungsprozess eine wichtige Rolle spielt. Während sogar eine schwere bronchiale Obstruktion oder ein deutlich erhöhter CRP-Spiegel allein das KHK-Risiko nur gering erhöht, führt die Kombination dieser beiden Faktoren zu einer stark erhöhten Infarktinzidenz.

Außerdem findet sich eine COPD bei Patienten mit einem metabolischen Syndrom vor allem dann, wenn der CRPSpiegel erhöht ist. Darüber hinaus sind die Entzündungsparameter bei Patienten mit geringer körperlicher Aktivität deutlich höher als bei solchen mit regelmäßiger sportlicher Betätigung. Umgekehrt konnte man in Studien zeigen, dass eine regelmäßige körperliche Akti- vität antiinflammatorisch wirkt. Einer neuen Studie zufolge dürften auch Statine bei COPD-Patienten unabhängig von dem Effekt auf das LDL-Cholesterin den Entzündungsprozess hemmen und somit die Prognose quoad vitam verbessern.

\section{Systemische Steroide nicht länger als zwei Wochen}

Für die Therapie der COPD stehen neben systemischen Steroiden auch inhalative Steroide, inhalative Anticholinergika und Beta-2-Sympathikomimetika zur Verfügung. Von praktischer Relevanz ist die Frage, inwieweit der Glukosestoffwechsel durch diese Substanzen beeinflusst wird.

„Bei systemischer Gabe von Glukokortikoiden kann es bereits nach einer zweiwöchigen Therapie zu behandlungsbedürftigen Hyperglykämien kommen", so Prof. Heinrich Worth, Fürth. Eine längere Therapie mit einer solchen weder der Krankheitsverlauf noch die Krankenhausverweildauer günstig beeinflusst wird. Neben den Auswirkungen auf den Glukosestoffwechsel müssten auch die Nebenwirkungen am Knochen und an der Muskulatur bedacht werden.

Doch wie sieht es mit den metabolischen Begleitwirkungen bei den inhalativen Kortikoiden aus? Laut Worth ist bei einer üblichen Dosierung nicht mit einem erhöhten Diabetesrisiko zu rechnen. Doch bei höherer Dosierung kann es zu einem Anstieg der Blutzuckerwerte bzw. des $\mathrm{HbA}_{1 c}$-Werts kommen. Substanz ist nicht vertretbar, da dadurch

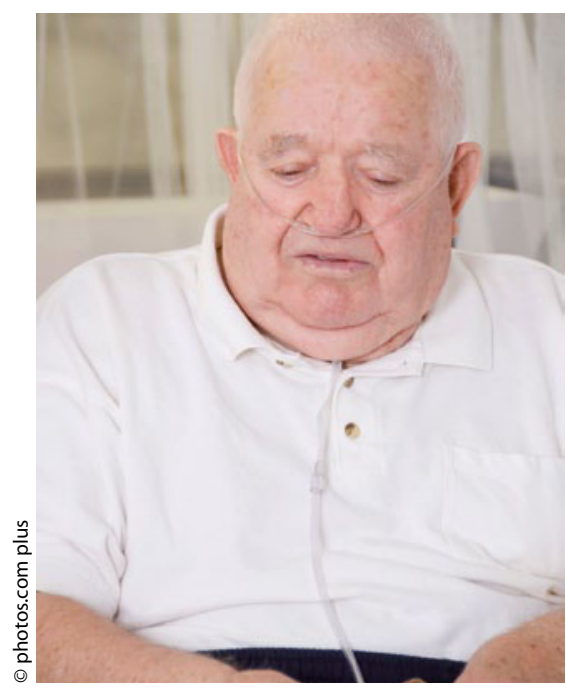

Cave: Die COPD-Medikation könnte eine Blutzuckerentgleisung hervorrufen.

\section{Verbessert Roflumilast die Insulinsensitivität?}

Bei den Bronchodilatatoren gibt es jedoch relevante Unterschiede. Während bei der Gabe eines Anticholinergikums nicht mit einer Blutzuckerentgleisung gerechnet werden muss, können sowohl die kurz- als auch die lang wirksamen Beta-2-Sympathikomimetika den Blutzucker signifikant erhöhen. Dagegen sind Theophyllin und das beim Asthma bronchiale eingesetzte Montelukast metabolisch neutral.

Eine neue Therapieoption für COPDPatienten ist der PDE-4-Hemmer Roflumilast $\left(\right.$ Daxas $\left.^{\circledast}\right)$. Erste Verlaufsbeobachtungen haben gezeigt, dass es nach einer zwölfwöchigen Therapie mit dieser Subs$\operatorname{tanz}$ zu einer Verbesserung der Stoffwechselsituation kommt. „Auch wenn der Mechanismus noch nicht genau bekannt ist, spricht vieles dafür, dass durch diese Substanz die Insulinsensitivität verbessert wird“, so Worth.

- Quelle: Kongress: „, Diabetologie grenzenlos“, 11.02.2011 in München 